\title{
Automated Border Control Systems: Biometric Challenges and Research Trends
}

\author{
Ruggero Donida Labati, Angelo Genovese, Enrique Muñoz, \\ Vincenzo Piuri, Fabio Scotti, and Gianluca Sforza \\ Department of Computer Science - Università degli Studi di Milano \\ Via Bramante 65, 26013 Crema, Italy \\ firstname. lastname@unimi.it
}

\begin{abstract}
Automated Border Control (ABC) systems automatically verify the travelers' identity using their biometric information, without the need of a manual check, by comparing the data stored in the electronic document (e.g., the e-Passport) with a live sample captured during the crossing of the border. In this paper, the hardware and software components of the biometric systems used in ABC systems are described, along with the latest challenges and research trends.
\end{abstract}

\section{Introduction}

The number of travelers in the world is constantly increasing [12] and International Border Crossing Points (BCP) are required to increase the passenger throughput, without sacrificing security or comfort. In this context, Automated Border Control (ABC) and surveillance systems can be deployed for an automatic, secure, fast, and user-friendly crossing procedure [51, 5, 50,60].

ABC systems, or e-Gates, typically include three steps: i) the document (e.g., the e-Passport) is checked for authenticity; ii) the identity of the traveler is verified based on his biometric traits; iii) the validity of the traveler authorization (e.g., the visa) is checked. Face and fingerprint recognition techniques are used in most of the e-Gates, with some systems using also the iris. If the biometric recognition is not successful, a manual check of the traveler identity is performed [23].

Three types of automated border crossing procedures are possible: i) one-step process; ii) integrated two-step process; iii) segregated two-step process [50]. In a one-step process, the document, the identity, and the authorization are verified at the same time, inside the e-Gate. In an integrated two-step process, the validity of the document is checked before letting the traveler go inside the e-Gate for the identity verification and for checking his travel authorization. In a segregated two-step process, the validity of the document and the travel authorization can be checked also at a different time and place of the border crossing.

In order to perform the required steps, four subsystems are used: i) the Document Authentication System (DAS), which checks the validity of the document; ii) the Biometric Verification System (BVS), which captures live biometric samples and compares them with the ones contained in the document; iii) the Central 
Systems Interface (CSI), which handles communication with external systems; iv) the Border Guard Maintenance System (BGMS), which is used by the officers to monitor the $\mathrm{ABC}$ system.

In order to check if the traveler is authorized for passage across the border, the ABC system checks with three external systems: i) the Visa Management Systems (VMS), which contains the visa information [46]; ii) the Registered Traveler Program (RTP), which contains the personal and biometric data of frequent travelers who voluntarily enrolled in the program; iii) the Entry-Exit Management Systems (EEMS), which contains the information about which borders the travelers cross, in order to detect overstayers, illegal immigration, and collect statistical information. In particular, the EU is proposing to officially adopt the RTP and EES in the ABC systems $[44,45,3,2]$.

\section{Biometric verification in $\mathrm{ABC}$ systems}

This section describes the biometric verification procedures using the face, the fingerprint, and the iris, which are the biometric traits used in ABC systems, as recommended by the ICAO [61]:

- Face recognition is the primary biometric trait adopted in e-Gates [61], since it is socially accepted, non-intrusive, and does not require special training. The biometric face verification consists of six steps: i) the system chooses the camera position based on the traveler's height; ii) information is displayed to instruct the traveler about how to position its head; iii) illumination is automatically adjusted based on environmental lights; iv) the face image is captured; v) a quality assessment module is used to determine if the image complies with the ISO recommendations [64,61]; vi) the matching between the live image and the sample in the document is performed.

- Fingerprint recognition is an optional biometric technology in e-Passports and e-Gates [61], features high recognition performances and good social acceptance, and is widely adopted. The biometric fingerprint verification consists of four steps: i) information is displayed to instruct the traveler about how to position the finger on the sensor; ii) the fingerprint image is captured; iii) a quality assessment module is used to determine if the image meets the required ISO recommendations [63]; iv) the matching between the live image and the sample contained in the document/database is performed. Minutiae-based matching algorithms are the most widespread $[67,55,58,72]$. Moreover, second generation European e-Passports store both face and fingerprint traits, which can be combined to increase the recognition accuracy $[74,22,4]$.

- Iris recognition is optional in e-Gates [61] and, while featuring very high recognition performances, is intrusive and has limited social acceptance, and for these reasons is not widely adopted. The biometric iris verification consists of four steps: i) information is displayed to instruct the traveler about where to place his head near the camera; ii) a near-infrared light pulse is 
used to illuminate the eye, as well as control the gaze direction and the dilation of the pupil; iii) the iris image is captured; iv) the live image and the sample contained in the document/database are matched.

\section{Challenges}

The most important challenges in the design of ABC systems regard the development of better anti-spoofing techniques, compatibility between systems, scalability of biometric systems, and methods for allowing the use of the e-Gates also to people with reduced mobility and visual impairments. Moreover, other challenges regard the capture of higher quality face and fingerprint images, and the design of less-intrusive iris biometric recognition technologies:

- Better anti-spoofing techniques, in particular liveness-based methods, are important to avoid cheating attempts that use, for example, printed face images [69], fake fingers made with silicone [68], or synthetic irises [14]. Recent projects studied enhanced anti-spoofing techniques for biometric systems [1], however the data about impostors trying to gain authorized access in e-Gates are not publicly available.

- Compatibility between systems should be realized by adopting a common biometric data format $[64,63,65]$, in order to facilitate the adoption of $\mathrm{ABC}$ systems. The type of data exchanged (sample or template) must be chosen according to bandwidth and privacy requirements $[79,40,20,16,8,11,7,17$, 21]. Moreover, a common standard for cryptographic interoperability could help the widespread adoption of security and privacy protection techniques [40].

- Scalable biometric systems must be designed, so that ABC systems are able to work efficiently on a large scale $[57,56]$.

- The design for people with reduced mobility and visual impairments could help people in a wheelchair, with muscular dystrophy, or with walking aids in accessing the e-Gate and interacting with the biometric sensors. Similarly, it could help visually impaired people when they can not see the information displayed to instruct them about the correct procedures.

- Higher quality face images greatly increase the recognition performances, but require the users to stand looking directly in front of the camera, which must be placed at the correct height. Moreover, the illumination must be uniform and able to compensate for environmental variations [78].

- Higher quality fingerprint images also increase the performance of fingerprint recognition technologies, and can be obtained by enhancing both the usability of the system and the algorithms for the quality estimation $[28,38$, $24]$, without increasing the acquisition time.

- Less-intrusive iris recognition techniques could help in extending the field of use iris-based systems, since they are currently the most accurate, but have high costs and intrusiveness. At the moment, iris recognition systems are not considered in e-Passports [71] and require additional systems for their use. 


\section{Research trends}

The most promising research trends in the design of innovative $\mathrm{ABC}$ systems regard the use of multibiometrics and less-constrained recognition:

- Multibiometrics can increase biometric recognition accuracy, usability, and robustness to spoofing attacks, by combining multiple biometric sources [74, 80]. Several studies demonstrate the increase of accuracy fusing face and fingerprint biometrics [77], also in the case of ABC systems [22,62]. Moreover, the non-universality or low discriminative power of some biometric traits (e.g., soft biometrics) can be compensated by fusing multiple traits $[66,54,15,6,53,52]$, which can then be used in automated border control and surveillance [73]. However, multibiometric systems are bigger, more complex, and handle more sensitive data, thus requiring more robust data protection schemes $[18,19]$.

- Less-constrained recognition could increase the usability and social acceptance of biometric systems [70]. In fact, since they allow a touchless recognition, it would be possible to perform the biometric verification at higher distances, with natural light conditions, and while the traveler is moving, by using the fingerprint [34, 35, 41, 33, 30, 29, 26, 39, 25,32], the palm [59], or the iris $[70,42,27,76,75,37]$. A study showed that touchless fingerprint technologies would be preferred over touch-based systems [35], thus allowing for an increased confidence and adoption of biometric recognition [43]. Moreover, less-constrained biometric recognition techniques using innovative traits are being researched $[13,36,31]$, and the advances in three-dimensional reconstruction techniques $[9,10,47-49]$ could allow the use of three-dimensional modeling methods for accurate, less-constrained biometric systems $[35,34$, $59,81]$.

\section{Conclusions}

The paper presented the biometric technologies adopted in ABC systems for the traveler's recognition, with a particular focus on the systems based on the face, the fingerprint, and the iris.

Moreover, the challenges of biometric systems in the context of ABC systems were discussed, with specific attention to their usability and to anti-spoofing techniques. The current issues of face, fingerprint, and iris recognition systems were also presented.

Lastly, the paper introduced the most promising research trends for a more accurate, usable, and socially accepted biometric recognition for travelers in ABC systems, with a specific focus on multibiometrics and less-constrained systems.

Acknowledgments This work was supported in part by: the EC within the 7FP under grant agreement 312797 (ABC4EU); the EC within the H2020 program under grant agreement 644597 (ESCUDO-CLOUD); and the Italian Ministry of Research within PRIN 2010-2011 project "GenData 2020" (2010RTFWBH). 


\section{References}

1. TABULA RASA - Trusted Biometrics under Spoofing Attacks - EU FP7 project (2010), https://www.tabularasa-euproject.org/

2. FastPass - a harmonized, modular reference system for all European automated border crossing points - EU FP7 project (2013), https://www.fastpass-project.eu

3. ABC4EU - Automated Border Controls for Europe - EU FP7 project (2014), http://abc4eu.com

4. Fiumicino is the first airport in Italy to automate border controls (2014), http://www.futuretravelexperience.com/2014/11/romes-fiumicino-airporttrialling-automated-border-control-e-gates

5. Amato, A., Di Lecce, V., Piuri, V.: Semantic Analysis and Understanding of Human Behavior in Video Streaming. SpringerLink : Bücher, Springer New York (2012)

6. Azzini, A., Marrara, S., Sassi, R., Scotti, F.: A fuzzy approach to multimodal biometric continuous authentication. Fuzzy Optimization and Decision Making 7(3), 215-302 (November 2008), 1568-4539

7. Barni, M., Bianchi, T., Catalano, D., Di Raimondo, M., Donida Labati, R., Failla, P., Fiore, D., Lazzeretti, R., Piuri, V., Scotti, F., Piva, A.: Privacy-preserving fingercode authentication. In: Proc. of the 2010 ACM Workshop on Multimedia and Security. pp. 231-240. New York, NY, USA (September 2010)

8. Barni, M., Bianchi, T., Catalano, D., Raimondo, M.D., Donida Labati, R., Failla, P., Fiore, D., Lazzeretti, R., Piuri, V., Scotti, F., Piva, A.: A privacy-compliant fingerprint recognition system based on homomorphic encryption and FingerCode templates. In: Proc. of the 2010 IEEE Int. Conf. on Biometrics: Theory Applications and Systems (BTAS). pp. 1-7. Washington, D.C., USA (September 2010)

9. Bellocchio, F., Borghese, N.A., Ferrari, S., Piuri, V.: 3D Surface Reconstruction: Multi-Scale Hierarchical Approaches. Springer (2013)

10. Bellocchio, F., Ferrari, S., Piuri, V., Borghese, N.A.: Hierarchical approach for multiscale support vector regression. IEEE Trans. on Neural Networks and Learning Systems 23(9), 1448-1460 (September 2012)

11. Bianchi, T., Donida Labati, R., Piuri, V., Piva, A., Scotti, F., Turchi, S.: Implementing FingerCode-based identity matching in the encrypted domain. In: Proc. of the 2010 IEEE Workshop on Biometric Measurements and Systems for Security and Medical Applications (BioMS). pp. 15-21. Taranto, Italy (September 2010)

12. Boeing: Current market outlook: 2014-2033 (2014), http://www.boeing.com

13. Bonissi, A., Donida Labati, R., Perico, L., Sassi, R., Scotti, F., Sparagino, L.: A preliminary study on continuous authentication methods for photoplethysmographic biometrics. In: Proc. of the 2013 IEEE Workshop on Biometric Measurements and Systems for Security and Medical Applications (BioMS). pp. 28-33. Napoli, Italy (September 2013)

14. Burge, M.J., Bowyer, K.: Handbook of iris recognition. Springer Science \& Business Media (2013)

15. Cimato, S., Gamassi, M., Piuri, V., Sana, D., Sassi, R., Scotti, F.: Personal identification and verification using multimodal biometric data. In: Proc. of the 2006 IEEE Int. Conf. on Computational Intelligence for Homeland Security and Personal Safety (CIHSPS). pp. 41-45. Alexandria, VA, USA (October 2006)

16. Cimato, S., Gamassi, M., Piuri, V., Sassi, R., Scotti, F.: Privacy issues in biometric identification. Touch Briefings pp. 40-42 (2006) 
17. Cimato, S., Gamassi, M., Piuri, V., Sassi, R., Scotti, F.: A biometric verification system addressing privacy concerns. In: Proc. of the 2007 Int. Conf. on Computational Intelligence and Security (CIS). pp. 594-598. Harbin, China (December 2007)

18. Cimato, S., Gamassi, M., Piuri, V., Sassi, R., Scotti, F.: Privacy-aware biometrics: design and implementation of a multimodal verification system. In: Proc. of the 2008 Annual Computer Security Applications Conf. (ACSAC). pp. 130-139. Anaheim, CA, USA (December 2008)

19. Cimato, S., Gamassi, M., Piuri, V., Sassi, R., Scotti, F.: A multi-biometric verification system for the privacy protection of iris templates. In: Corchado, E., Zunino, R., Gastaldo, P., Herrero, A. (eds.) Proc. of the Int. Workshop on Computational Intelligence in Security for Information Systems (CISIS), Advances in Soft Computing, vol. 53, pp. 227-234. Springer Berlin Heidelberg (2009)

20. Cimato, S., Gamassi, M., Piuri, V., Sassi, R., Scotti, F.: Privacy in biometrics. In: Boulgouris, N.V., Plataniotis, K.N., Micheli-Tzanakou, E. (eds.) Biometrics: theory, methods, and applications, pp. 633-654. Computational Intelligence, WileyIEEE Press (2009)

21. Cimato, S., Sassi, R., Scotti, F.: Biometrics and privacy. Recent Patents on Computer Science 1, 98-109 (June 2008), 1874-4796

22. Cuesta Cantarero, D., Perez Herrero, D.A., Martin Mendez, F.: A multi-modal biometric fusion implementation for ABC systems. In: Proc. of the 2013 European Intelligence and Security Informatics Conf. (EISIC). pp. 277-280. Uppsala, Sweden (August 2013)

23. Donida Labati, R., Genovese, A., Muñoz, E., Piuri, V., Scotti, F., Sforza, G.: Advanced design of automated border control gates: biometric system techniques and research trends. In: Proc. of the 2015 IEEE Int. Symp. on Systems Engineering (ISSE). Rome, Italy (September 2015)

24. Donida Labati, R., Genovese, A., Muñoz, E., Piuri, V., Scotti, F., Sforza, G.: Automatic classification of acquisition problems affecting fingerprint images in automated border controls. In: Proc. of the 2015 IEEE Symp. on Computational Intelligence in Biometrics and Identity Management (CIBIM '15). Cape Town, South Africa (December 2015)

25. Donida Labati, R., Genovese, A., Piuri, V., Scotti, F.: Measurement of the principal singular point in contact and contactless fingerprint images by using computational intelligence techniques. In: Proc. of the 2010 IEEE Int. Conf. on Computational Intelligence for Measurement Systems and Applications (CIMSA). pp. 18-23. Taranto, Italy (September 2010)

26. Donida Labati, R., Genovese, A., Piuri, V., Scotti, F.: Fast 3-D fingertip reconstruction using a single two-view structured light acquisition. In: Proc. of the 2011 IEEE Workshop on Biometric Measurements and Systems for Security and Medical Applications (BioMS). pp. 1-8. Milan, Italy (September 2011), 978-1-4577-0765-0

27. Donida Labati, R., Genovese, A., Piuri, V., Scotti, F.: Iris segmentation: state of the art and innovative methods. In: Liu, C., Mago, V. (eds.) Cross Disciplinary Biometric Systems, Intelligent Systems Reference Library, vol. 37, pp. 151-182. Springer (2012)

28. Donida Labati, R., Genovese, A., Piuri, V., Scotti, F.: Quality measurement of unwrapped three-dimensional fingerprints: a neural networks approach. In: Proc. of the 2012 IEEE-INNS Int. Joint Conf. on Neural Networks (IJCNN). pp. 11231130. Brisbane, Australia (June 2012) 
29. Donida Labati, R., Genovese, A., Piuri, V., Scotti, F.: Two-view contactless fingerprint acquisition systems: a case study for clay artworks. In: Proc. of the 2012 IEEE Workshop on Biometric Measurements and Systems for Security and Medical Applications (BioMS). pp. 1-8. Salerno, Italy (September 2012)

30. Donida Labati, R., Genovese, A., Piuri, V., Scotti, F.: Virtual environment for 3-D synthetic fingerprints. In: Proc. of the 2012 IEEE Int. Conf. on Virtual Environments, Human-Computer Interfaces and Measurement Systems (VECIMS). pp. 48-53. Tianjin, China (July 2012)

31. Donida Labati, R., Genovese, A., Piuri, V., Scotti, F.: Weight estimation from frame sequences using computational intelligence techniques. In: Proc. of the 2012 IEEE Int. Conf. on Computational Intelligence for Measurement Systems and Applications (CIMSA). pp. 29-34. Tianjin, China (July 2012)

32. Donida Labati, R., Genovese, A., Piuri, V., Scotti, F.: Accurate 3D fingerprint virtual environment for biometric technology evaluations and experiment design. In: Proc. of the 2013 IEEE Int. Conf. on Computational Intelligence and Virtual Environments for Measurement Systems and Applications (CIVEMSA). pp. 43-48. Milan, Italy (July 2013)

33. Donida Labati, R., Genovese, A., Piuri, V., Scotti, F.: Contactless fingerprint recognition: a neural approach for perspective and rotation effects reduction. In: Proc. of the IEEE Workshop on Computational Intelligence in Biometrics and Identity Management (CIBIM). pp. 22-30. Singapore, Singapore (April 2013)

34. Donida Labati, R., Genovese, A., Piuri, V., Scotti, F.: Touchless fingerprint biometrics: a survey on 2D and 3D technologies. Journal of Internet Technology 15(3), 325-332 (2014)

35. Donida Labati, R., Genovese, A., Piuri, V., Scotti, F.: Toward unconstrained fingerprint recognition: a fully-touchless 3-D system based on two views on the move. IEEE Trans. on Systems, Man, and Cybernetics: Systems (2015)

36. Donida Labati, R., Piuri, V., Sassi, R., Sforza, G., Scotti, F.: Adaptive ECG biometric recognition: a study on re-enrollment methods for QRS signals. In: Proc. of the IEEE Workshop on Computational Intelligence in Biometrics and Identity Management (CIBIM). pp. 30-37. Orlando, FL, USA (December 2014)

37. Donida Labati, R., Piuri, V., Scotti, F.: Neural-based iterative approach for iris detection in iris recognition systems. In: Proc. of the IEEE Symp. on Computational Intelligence for Security and Defence Applications (CISDA). pp. 1-6. Ottawa, Canada (July 2009)

38. Donida Labati, R., Piuri, V., Scotti, F.: Neural-based quality measurement of fingerprint images in contactless biometric systems. In: Proc. of the 2010 IEEE-INNS Int. Joint Conf. on Neural Networks (IJCNN). pp. 1-8. Barcelona, Spain (July 2010)

39. Donida Labati, R., Piuri, V., Scotti, F.: A neural-based minutiae pair identification method for touchless fingeprint images. In: Proc. of the 2011 IEEE Workshop on Computational Intelligence in Biometrics and Identity Management (CIBIM). pp. 96-102. Paris, France (April 2011)

40. Donida Labati, R., Piuri, V., Scotti, F.: Biometric privacy protection: guidelines and technologies. In: Obaidat, M.S., Sevillano, J.S., Joaquim, F. (eds.) Communications in Computer and Information Science, vol. 314, pp. 3-19. Springer (2012)

41. Donida Labati, R., Piuri, V., Scotti, F.: Touchless Fingerprint Biometrics. Security, Privacy and Trust, CRC Press (2015)

42. Donida Labati, R., Scotti, F.: Noisy iris segmentation with boundary regularization and reflections removal. Image and Vision Computing, Special Issue on Iris Images Segmentation 28(2), 270-277 (2010) 
43. El-Abed, M., Giot, R., Hemery, B., Rosenberger, C.: A study of users' acceptance and satisfaction of biometric systems. In: Proc. of the 2010 IEEE Int. Carnahan Conf. on Security Technology (ICCST). pp. 170-178. San Jose, CA, USA (October 2010)

44. European Commission: Proposal for a regulation of the European Parliament and of the Council establishing a Registered Traveller Programme (2013), http://ec.europa.eu

45. European Commission: Proposal for a regulation of the European Parliament and of the Council establishing an Entry/Exit System (EES) to register entry and exit data of third country nationals crossing the external borders of the Member States of the European Union (2013)

46. European Parliament: Regulation (EC) No. 767/2008 of the European Parliament and of the Council of 9 July 2008 concerning the Visa Information System (VIS) and the exchange of data between Member States on short-stay visas (2008)

47. Ferrari, S., Bellocchio, F., Piuri, V., Alberto Borghese, N.A.: A hierarchical RBF online learning algorithm for real-time 3-D scanner. IEEE Trans. on Neural Networks 21(2), 275-285 (February 2010)

48. Ferrari, S., Ferrigno, G., Piuri, V., Alberto Borghese, N.A.: Reducing and filtering point clouds with enhanced vector quantization. IEEE Trans. on Neural Networks 18(1), 161-177 (January 2007)

49. Ferrari, S., Frosio, I., Piuri, V., Alberto Borghese, N.: Automatic multiscale meshing through HRBF networks. IEEE Trans. on Instrumentation and Measurement 54(4), 1463-1470 (August 2005)

50. Frontex Agency: Best practice operational guidelines for automated border control (ABC) systems. Tech. rep., European Agency for the Management of Operational Cooperation at the External Borders of the Member States of the European Union (2012), http://frontex.europa.eu

51. Frontex Agency: Best practice technical guidelines for automated border control (ABC) systems. Tech. rep., European Agency for the Management of Operational Cooperation at the External Borders of the Member States of the European Union (2012), http://frontex.europa.eu

52. Gamassi, M., Lazzaroni, M., Misino, M., Piuri, V., Sana, D., Scotti, F.: Accuracy and performance of biometric systems. In: Proc. of the 2004 IEEE Instrumentation and Measurement Technology Conf. (IMTC). pp. 510-515. Como, Italy (May 2004)

53. Gamassi, M., Lazzaroni, M., Misino, M., Piuri, V., Sana, D., Scotti, F.: Quality assessment of biometric systems: a comprehensive perspective based on accuracy and performance measurement. IEEE Trans. on Instrumentation and Measurement 54(4), 1489-1496 (2005)

54. Gamassi, M., Piuri, V., Sana, D., Scotti, F.: A high-level optimum design methodology for multimodal biometric systems. In: Proc. of the 2004 IEEE Int. Conf. on Computational Intelligence for Homeland Security and Personal Safety (CHISPS). pp. 117-124. Venice, Italy (July 2004)

55. Gamassi, M., Piuri, V., Sana, D., Scotti, F.: Robust fingerprint detection for access control. In: Proc. of the Workshop RoboCare. CNR, Rome, Italy (May 2005)

56. Gamassi, M., Piuri, V., Sana, D., Scotti, F., Scotti, O.: Scalable distributed biometric systems - advanced techniques for security and safety. IEEE Instrumentation \& Measurement Mag. 9(2), 21-28 (April 2006)

57. Gamassi, M., Piuri, V., Sana, D., Scotti, O., Scotti, F.: A multi-modal multiparadigm agent-based approach to design scalable distributed biometric systems. In: Proc. of the 2005 IEEE Int. Conf. on Computational Intelligence for Homeland Security and Personal Safety (CIHSPS). pp. 65-70. Orlando, FL, USA (April 2005) 
58. Gamassi, M., Piuri, V., Scotti, F.: Fingerprint local analysis for high-performance minutiae extraction. In: Proc. of the 2005 IEEE Int. Conf. on Image Processing (ICIP). vol. 3, pp. 265-268. Genoa, Italy (September 2005)

59. Genovese, A., Piuri, V., Scotti, F.: Touchless Palmprint Recognition Systems, Advances in Information Security, vol. 60. Springer (2014)

60. Gorodnichy, D., Yanushkevich, S., Shmerko, V.: Automated border control: Problem formalization. In: Proc. of the 2014 IEEE Symp. on Computational Intelligence in Biometrics and Identity Management (CIBIM). pp. 118-125. Orlando, FL, USA (December 2014)

61. International Civil Aviation Organization: Doc 9303, machine readable travel documents. Part 1, vol. 2 (2006), http://www.icao.int/publications/pages/publication.aspx?docnum $=9303$

62. International Organization for Standardization (ISO: ISO/IEC 24722:2007, Information technology - Biometrics - Multimodal and other multibiometric fusion (2007)

63. International Organization for Standardization (ISO): ISO/IEC 19794-4:2011, Information technology - Biometric data interchange formats - part 4: Finger image data (2011)

64. International Organization for Standardization (ISO): ISO/IEC 19794-5:2011, Information technology - Biometric data interchange formats - part 5: Face image data (2011)

65. International Organization for Standardization (ISO): ISO/IEC 19794-6:2011, Information technology - Biometric data interchange formats - part 6: Iris image data (2011)

66. Jain, A.K., Ross, A.: Multibiometric systems. Communications of the ACM 47(1), 34-40 (2004)

67. Maltoni, D., Maio, D., Jain, A.K., Prabhakar, S.: Handbook of fingerprint recognition. Springer, second edn. (2009)

68. Marasco, E., Ross, A.: A survey on antispoofing schemes for fingerprint recognition systems. ACM Computing Surveys 47(2), 1-36 (2014)

69. Marcel, S., Nixon, M.S., Li, S.Z.: Handbook of Biometric Anti-spoofing: Trusted Biometrics Under Spoofing Attacks. Advances in computer vision and pattern recognition, Springer London, Limited (2014)

70. Matey, J.R., Naroditsky, O., Hanna, K., Kolczynski, R., LoIacono, D.J., Mangru, S., Tinker, M., Zappia, T.M., Zhao, W.Y.: Iris on the move: Acquisition of images for iris recognition in less constrained environments. Proc. of the IEEE 94(11), 1936-1947 (November 2006)

71. Palmer, A.J., Hurrey, C.: Ten reasons why IRIS needed 20:20 foresight: some lessons for introducing biometric border control systems. In: Proc. of the 2012 European Intelligence and Security Informatics Conf. (EISIC). pp. 311-316. Odense, Denmark (2012)

72. Piuri, V., Scotti, F.: Fingerprint biometrics via low-cost sensors and webcams. In: Proc. of the 2008 IEEE Int. Conf. on Biometrics: Theory, Applications and Systems (BTAS). pp. 1-6. Washington, D.C., USA (September 2008)

73. Reid, D., Samangooei, S., Chen, C., Nixon, M.S., Ross, A.: Soft biometrics for surveillance: an overview. In: Machine Learning: Theory and Applications, pp. 327-352. 31, Elsevier (2013)

74. Ross, A., Nandakumar, K., Jain, A.: Handbook of Multibiometrics, International Series on Biometrics, vol. 6. Springer (2006) 
75. Scotti, F.: Computational intelligence techniques for reflections identification in iris biometric images. In: Proc. of the 2007 IEEE Int. Conf. on Computational Intelligence for Measurement Systems and Applications (CIMSA). pp. 84-88. Ostuni, Italy (June 2007)

76. Scotti, F., Piuri, V.: Adaptive reflection detection and location in iris biometric images by using computational intelligence techniques. IEEE Trans. on Instrumentation and Measurement 59(7), 1825-1833 (July 2010)

77. Snelick, R., Indovina, M., Yen, J., Mink, A.: Multimodal biometrics: Issues in design and testing. In: Proc. of the ACM International Conference on Multimodal Interfaces (ICMI). pp. 68-72. Vancouver B.C., Canada (November 2003)

78. Spreeuwers, L.J., Hendrikse, A.J., Gerritsen, K.J.: Evaluation of automatic face recognition for automatic border control on actual data recorded of travellers at Schiphol Airport. In: Proc. of the Int. Conf. of the Biometrics Special Interest Group (BIOSIG). pp. 1-6. Darmstadt, Germany (September 2012)

79. Unisys: Entry-exit feasibility study - final report. Tech. rep., European Commission (2008), http://www.europarl.europa.eu

80. Wei, H., Chen, L., Ferryman, J.: Biometrics in ABC: Counter-spoofing research. In: Proc. of the Frontex Global Conference on Future Developments of Automated Border Control (ABC). Warsaw, Poland (2013)

81. Zhang, D., Lu, G.: 3D Biometrics: Systems and Applications. Springer Publishing Company, Incorporated (2013) 